\title{
Philosophiques
}

\section{Un nouveau paradigme : le corps sportif (suite)}

\section{Léo-Paul Bordeleau}

Volume 12, numéro 2, automne 1985

URI : https://id.erudit.org/iderudit/203288ar

DOI : https://doi.org/10.7202/203288ar

Aller au sommaire du numéro

Éditeur(s)

Société de philosophie du Québec

ISSN

0316-2923 (imprimé)

1492-1391 (numérique)

Découvrir la revue

Citer cet article

Bordeleau, L.-P. (1985). Un nouveau paradigme : le corps sportif (suite).

Philosophiques, 12(2), 247-279. https://doi.org/10.7202/203288ar d'utilisation que vous pouvez consulter en ligne.

https://apropos.erudit.org/fr/usagers/politique-dutilisation/ 


\title{
ARTICLES
}

\section{UN NOUVEAU PARADIGME : LE CORPS SPORTIF (suite)}

\author{
par Léo-Paul Bordeleau
}

\section{B. UNE SPORTIVISATION DU CORPS}

Le corps humain nous est apparu comme une réalité plurielle dont chaque élément ne saurait épuiser le mot même qui coiffe chacun d'eux. Selon notre interprétation qui, faut-il le mentionner, interroge une longue histoire du dualisme corpsâme, le corps n'est plus réductible au physique ; il traduit plutôt un ensemble organisé de structures qui, lorsqu'elles se rapportent à l'individu humain en tant que tel, sont des structures anatomo-physiologique, libidinale et fantasmée, sociale et mythique ${ }^{1}$.

Or, il nous apparaît que, depuis l'avènement de la société industrielle, c'est-à-dire le moment où le corps réprimé fut remis dans l'ordre des choses par une fragmentation de ses potentialités selon une logique plus discrète ou plus rusée du pouvoir de la raison, c'est en tant que réalité plurielle que le corps a mieux servi les objectifs de la raison. Toutefois, comme pour mieux conjurer ou exorciser ce qui, de la corporéité, contribue encore à brouiller sa transparence et sa jouissance, la raison s'est engagée sur une voie nouvelle de contrôle par un usage, légitime cette fois, du corps ; autrement dit, plutôt que de l'oublier ou le mépriser au bénéfice de la contemplation, ou encore de l'abrutir par et dans le travail, elle l'exalte en s'aventurant elle-même

1. Nous avons développé ces idées dans la première partie (A) de cet article, publiée dans Philosophiques, avril 1985, pages 33-52. 
dans un lieu où le corps est en tous points privilégié, c'est-àdire le sport. Elle se fait ainsi sportive. Telle est, nous semble$\mathrm{t}$-il, la plus récente intervention d'une raison qui prétend dompter et récupérer le plus rebelle de ses obstacles. Or, nous prétendons que cette tentative de conjuration et de possession d'un corps humain vécu comme foyer de puissance et de pouvoir, de désirs et de plaisirs, d'agressivité et de violence, s'effectue en fabriquant et en lui substituant une doublure paradigmatique que nous appelons "le corps sportif ». C'est cette nouvelle démarche astucieuse de la raison que nous voulons retracer et démonter. C'est le processus de sportivisation du corps humain qui retiendra notre attention.

\section{Le sport comme système}

Le système sportif se présente aujourd'hui, dans la plupart des sociétés techno-industrielles, comme la forme dominante d'éducation du corps. Mais, au fait, pourquoi parle-t-on de système sportif ? En quoi le sport est-il un système ? Répondre à cette question suppose, d'abord, une prise de conscience du fait que le sport moderne est devenu un phénomène socio-culturel massif dont la prégnance et le poids d'évidence n'étonnent guère ${ }^{2}$; c'est aussi faire appel à une explication de la dimension institutionnelle et systématique du sport ; plus précisément encore, c'est tenter de montrer la manière dont le sport (la raison sportive) récupère, condense et contrôle la puissance physique, la charge de désir et d'agressivité, la teneur sociale et mythique du corps humain. Nous limitons notre étude à ces deux derniers points.

À vrai dire, désigner le sport comme institution et comme système reste une détermination à priori, dans la mesure où on

2. Cette dimension du phénomène sportif fait l'objet de quelques sciences sociales. Pourtant ce phénomène omniprésent, aux manifestations éclatantes, reste encore bien occulté aux yeux du chercheur socio-culturel. Si l'on dispose de données quantitatives considérables (enquêtes, statistiques, observations directes, entretiens, etc.) et si les études de pédagogie sportive ou de psychologie du sport sont relativement abondantes, on remarque par contre une très grande pénurie d'études critiques sur la signification et la valeur existentielle de ce phénomène, ainsi que sur les effets réels de masse, notamment les effets du spectacle sportif et de l'idéologie véhiculée par eux sur la conscience politique du spectateur, l'effet de catharsis de l'effort sportif sur les adolescents, l'effet de coexistence pacifique sportive sur la vie quotidienne des citoyens, l'effet de la violence spectacularisée, etc.. 
ne dévoile pas la postulation d'un modèle institutionnel et d'un système référentiel. Il importe donc de préciser les conditions de possibilité de l'institution et du système proprement dits, pour voir ensuite si et en quel sens la réalité sportive les reproduit.

Plutôt que de nous interroger sur la genèse et le développement des concepts d'institution et de système, nous préférons nous laisser inspirer par l'enseignement étymologique et l'analyse socio-culturelle, et ainsi tenter de dire ce que ces concepts réalisés sont pour nous. Les termes "institution " et " système " traduisent l'idée d'organisation, de stabilisation et d'enchaînement. Dans le domaine psycho-social, celui qui nous intéresse ici, il s'agit d'organisation de besoins, de désirs, d'initiatives, d'actes, de représentations, etc.. On constate en effet que les individus humains, consciemment ou non, construisent des normes et des formes de comportement, pour s'y référer et s'y soumettre, soit par souci de sécurité et d'efficacité, soit en vue d'une économie d'efforts dans leurs manières d'agir et de penser, soit pour faciliter la communication entre eux, ou encore en vue d'une meilleure prévision du comportement de l'autre. Si l'on pousse encore plus loin l'observation, on remarque que des groupes concrets, sans aucun lien intime les uns avec les autres, construisent et suivent également des modèles communs, qui varient d'une société à l'autre, mais qui, au sein d'une société donnée, s'imposent à tous ces groupes occupés à des fonctions semblables. Ainsi, l'institution est à la fois un modèle, une norme et une forme sociale. Elle naît d'un besoin et d'une volonté d'organiser et de structurer un ensemble d'éléments ou de fonctions, caractérisé par le souci d'une certaine permanence dans la durée, dans le but de réaliser des objectifs communs précis. Dès lors, institutionnaliser correspond à une manière formaliste de fonctionner, commandée par un souci de sécurité, de stabilité et d'efficacité ; cette manière est ordinairement constituée de règles, de lois, de codes, de statuts, de procédures, d'une certaine bureaucratie des rapports humains, de contrats et de rites. C'est donc dire que les dispositions psychologiques individuelles produisent les institutions ; mais en retour, le fait d'être rassemblé en institutions crée des conditionnements qui influencent le comportement de l'individu. Le processus d'institutionnalisa- 
tion est donc ambivalent : d'une part, il assure une stabilité suffisante, il donne une sécurité réelle aux rapports humains et il rend plus efficaces les échanges, y compris les tensions et les conflits qu'ils impliquent ; d'autre part, ce processus est essentiellement dynamique puisque les actions humaines et leurs règles ne sont ni comprises ni appliquées de la même façon à des moments successifs, alors même que les formules qui les expriment peuvent rester littéralement les mêmes.

En outre, les actions sociales et leurs règles ont été représentées avyec un souci d'intégration logique plus serré encore. C'est ainsi que, pour plus de stabilité, de contrôle et d'efficacité, on va chercher à inscrire les actions sociales, incluant leurs auteurs et leurs règles, à les ordonner même et à les hiérarchiser comme éléments fonctionnels dans un ensemble rationalisé et pourvu d'une logique propre : tel est le processus de systématisation ; de là les systèmes social, économique, politique, culturel et sportif. Du fait qu'il forme un ensemble organique, le système implique que ses éléments constitutifs aient des propriétés communes plutôt qu'uniques et qu'ils reçoivent un statut fonctionnel : tout système se caractérise par l'ordre entre ses éléments ; tout processus de systématisation contraint effectivement chaque élément du système à exercer une fonction logistique, donc à perdre sa valeur propre au profit d'une valeur fonctionnelle et d'une valeur de cohérence du système proprement dit. En somme, la notion de système ne renvoie pas tant à une chose qu'à un ordre de choses entre des phénomènes, des variables ou des parties, réduits au statut de l'« être-pour-l'ensemble ».

Dès lors, on peut dire qu'une réalité psycho-sociale devient institution et système dans ia mesure où, pour des raisons de stabilité, de sécurité, d'efficacité et de contrôle, ses éléments constitutifs entretiennent entre eux des rapports d'interdépendance et de fonctionnalité, dans la mesure aussi où la totalité formée par l'ensemble des éléments est irréductible à leur somme, et où les rapports d'interdépendance entre les éléments et la totalité sont régis par une logique systémique.

La réalité sportive moderne répond-elle à ces exigences ? On a déjà constaté que le sport moderne est un phénomène socioculturel prégnant et complexe. En effet, si on veut bien y regar- 
der de plus près, on s'aperçoit que la réalité sportive aujourd'hui couvre et intègre une pluralité de comportements plus ou moins codifiés et ritualisés, de représentations et de valeurs diversement inscrites dans des schèmes techniques et des formes culturelles variés. Ainsi, par exemple, grâce à des mécanismes de compensation et d'ajustement qu'il offre à l'effort physique et mental du travailleur, grâce à la croyance qu'il suscite en une autonomie et en un épanouissement individuels, grâce aussi à l'optimisme idéologique qui en résulte, le sport moderne, tant dans sa forme récréative que dans sa forme scolaire, est devenu à la fois un renforcement et une sorte de contrepartie du travail manuel et intellectuel. Il y a plus cependant. La mise en œuvre d'une organisation technocratique et bureaucratique de la réalité sportive dans ses formes économiques, industrielles, commerciales et financières, sa mise en forme spectaculaire avec, comme conséquences, sa valeur marchande et son utilisation culturelle et politique comme cérémonial et fête, la mobilisation des énergies de la masse spectatrice, ainsi que l'exacerbation et la canalisation de l'agressivité, ont fait du sport moderne un univers microscopique imposant qui étend ses tentacules partout où sont impliqués enjeux et agressivité, efficacité et performance, mesures et records.

Dès lors, la réalité sportive moderne se présente bien comme un ensemble structuré et organique, dynamique et historique, capable de produire et de transformer ses orientations et ses catégories de fonctionnement. Avec ses structures propres, sa hiérarchie, sa réglementation spécifique, ses techniques et ses installations, son personnel qualifié, sa lutte de classes pour le contrôle de son historicité, ses orientations culturelles, politiques et économiques, le sport moderne fonctionne comme une institution. En d'autres termes, le sport moderne est devenu une institution à partir du moment où le complexe de valeurs et de normes, de règles codifiées et garanties par un personnel officiel, de rôles et de rituels, de techniques et de technologie, de connaissances transmises, s'est organisé d'une façon spécifiquement sportive, par souci de stabilité, de sécurité, d'efficacité, et avec une certaine constance dans le temps ${ }^{3}$.

3. Le statut institutionnel du sport est relativement récent. C'est au cours de la première moitié du XIX ${ }^{\mathrm{e}}$ siècle, en Angleterre, que le sport a reçu la physionomie propre que nous lui reconnaissons aujourd'hui. En fait, le sport moderne est né de et dans l'institution 
L'envergure du sport moderne et sa vaste pénétration dans tous les domaines de la société font qu'on doit le considérer comme une institution sociale à part entière. C'est particulièrement vrai dans la mesure où il influence aussi bien lè monde des affaires que la mode et la publicité, le prestige social et le culte du héros. C'est en tant qu'institution que le sport moderne indique à ses adeptes (acteurs, spectateurs et imitateurs) comment ils doivent agir, se comporter, percevoir le monde qui les entoure et croire en des valeurs. En outre, dans la mesure où cette pratique et cette vision sportivisées du monde sont précises et stables, dans la mesure aussi où elles combinent structure sociale et structure culturelle, elles sont susceptibles d'être utilisées comme modèle d'organisation et d'efficacité. En ce sens, le sport moderne fait plus que refléter la société dans laquelle il existe ; il en est le référent.

Le sport moderne est aussi un système puisqu'il en réalise toutes les conditions d'existence. En effet, le sport moderne commande et ordonne les activités et les fantasmes du sportif (acteurs, spectateurs et imitateurs) selon des structures, un processus et une logique propres au groupe d'individus impliqués d'une manière ou d'une autre dans le sport. Autrement dit, dans la mesure où la stabilité, le contrôle et l'efficacité nécessitent la présence d'éléments ayant entre eux des rapports d'interdépendance (ici, action des corps, activités ludiques, luttes compétitives, règles et techniques, spectacle et complexe de mythes), dans la mesure où ces déterminations supposent un arrangement rationnel, ordonné et fonctionnel de tous ces éléments en une totalité visible mais différente de ses parties constitutives et des autres organisations collectives, le sport moderne tend non seulement à reproduire le système social qui l'a engendré, il devient lui-même un système. Dans la mesure où le sport moderne est un cadre standardisé à l'intérieur duquel les individus doivent s'adapter aux formes de travail qu'exigent la performance sportive et la compétition, dans la mesure aussi où il est une orga-

scolaire, à titre de moyen disciplinaire des corps et des volontés : il est alors une activité physique caractérisée par une compétition plus ou moins ludique dans le but de procurer une formation morale par une formation corporelle. L'avènement de l'industrialisme, avec ses principes de standardisation et de spécialisation, de synchronisation et de concentration, de maximalisation et de centralisation, a contribué à faire du sport moderne l'un des soussystèmes les plus puissants de l'ère techno-industrielle. 
nisation méthodique et efficace des activités sportives, avec son réseau administratif structuré visant ainsi à la meilleure intégration fonctionnelle des individus, on peut parler de système sportif. Dès lors, par sa logique spécifique, qui est une logique de l'effort rationnel et performatif des corps et par voie de conséquence, une logique uniformisatrice des consciences, pour plus de puissance, de succès, de prestige et de plaisir, le système sportif parvient à condenser et à concentrer tout ce qu'on retrouve dans le système social global.

L'avantage qu'il y a à considérer le sport moderne comme système est précieux ; il permet de percevoir les composantes du sport moderne comme des éléments fonctionnels, par conséquent, de les classifier selon le rôle de chacun, de mesurer leur performance par rapport à la logique et aux exigences de l'ensemble. C'est ainsi que, dans le système sportif, il y a, par exemple, des producteurs et des consommateurs : les producteurs sont les joueurs, les capitaines d'équipes, les entraîneurs, les administrateurs et autres membres du personnel responsable de l'organisation des manifestations sportives; les consommateurs sont les spectateurs, les téléspectateurs, les auditeurs de la radio, les lecteurs de journaux, ou tout simplement les gens qui parlent de sport entre eux.

Cette représentation du sport comme système ne doit pas cependant nous tromper et nous laisser croire en son indépendance par rapport aux autres systèmes, comme le suggèrent multiples définitions proposées. Le sport a une histoire et il est dans l'histoire. En tant que réalité socio-culturelle, il ne peut exister que par son action sur lui-même, conjuguée à celle des autres organisations sociales : il est ce qu'en font les agents sportifs en inter-relation avec les agents sociaux en général. Ainsi, il n'y a pas de système sportif définissable indépendamment des acteurs sociaux multiples et de leurs orientations particulières. On ne saurait, par conséquent, concevoir le sport moderne à la manière d'un système clos sur lui-même, comme bien des puristes le voudraient ; c'est en réalité un système en constantes interrelations fonctionnelles avec d'autres systèmes socio-culturels, mais véhiculant à sa manière des objectifs similaires et les pénétrant tous de son dynamisme et de son esprit. 
Or, c'est en tant qu'institution et système qu'à notre avis le sport moderne se présente comme une forme dominante d'éducation des corps. C'est une raison d'institution et de système, devenue sportive, qui s'emploie à la fabrication même du corps sportif, ce nouveau modèle de perception et d'action des corps et partant des individus eux-mêmes. Telle est l'idée que nous voulons développer en centrant notre analyse sur quatre points :

- le sport comme processus de rationalisation des corps et des individus, dans la mesure où le corps est perçu comme une structure anatomo-physiologique et utilisé sous un mode machinique ;

- le sport comme systématisation de l'instinct agonal, dans la mesure où le corps est représenté comme matière désirante et foyer privilégié d'agressivité ;

- le sport comme hiérarchisation des corps et des individus, en fonction de leurs aptitudes performatives ;

- le sport comme système de mythes, dans la mesure où il mobilise et exploite, par son spectacle, l'imaginaire individuel et collectif.

\section{Le sport comme processus de rationalisation des corps}

Le sport moderne se développe comme le plus rigoureux des processus de rationalisation du geste naturel et des corps, et ceci pour au moins deux raisons : d'une part, parce que la rationalité technologique constitue la base même du progrès sportif et fournit le modèle d'esprit et de comportement pour toutes sortes de réalisations productives de cet ordre; d'autre part, parce que le corps humain a été représenté d'une façon privilégiée comme une structure anatomo-physiologique, et par là même soumis quasi exclusivement aux lois de la mécanique, à une parcellisation de ses tâches pour plus de contrôle et d'efficacité, donc perçu et utilisé sous un mode machinique.

Nous avons déjà souligné que, dans notre système socioéconomique, le concept de production acquiert une détermination nouvelle, en ce sens que la production vise essentiellement l'échange. Or, il ne fait plus de doute que, dans l'actuelle civilisation, le développement croissant de cette production est lié à celui du machinisme techno-industriel, dont la mécanisation, 
la division et l'organisation des tâches de production ont contribué à créer un temps chronométrique du travail devenu le cadre de toute activité rentable. Toute la production industrialiste est ainsi devenue une course au rendement par l'amélioration de la technique.

On retrouve ce dynamisme intégralement ancré dans le sport moderne, dans la mesure où celui-ci fait partie intégrante de notre civilisation technicienne : les rapports entre le progrès technique et l'accroissement du sport moderne sont réels et obvies. C'est pourquoi la recherche et le progrès des performances sportives ont amené les théoriciens et les producteurs du sport moderne à tayloriser la force sportive, tant physique que psychique : pour être rentables, le geste et le mouvement sportifs doivent être économiques, par conséquent rationnellement déterminés.

Or, cette technicisation du geste et du mouvement n'est possible que dans la mesure où le corps biologique est perçu et utilisé comme une machine. La représentation' machinique du corps est particulièrement favorisée par le discours imposant de l'anatomie et de la physiologie. Ainsi, par exemple, A. LEROY et J. VIVES conçoivent le corps comme une machine humaine qu'ils étudient sous les rapports de la mécanique et de la physiologie :

Nous considérons le corps humain à la fois : - comme un corps quelconque, soumis par conséquent aux lois générales de la physique et jouissant des propriétés communes aux corps (compressibilité, élasticité, mobilité, pesanteur, inertie, etc.) ;-comme une machine, c'est-à-dire comme un système de corps, d'organes (. . .) destinés à transmettre l'action des forces, à faire agir une puissance sur une résistance et capables d'engendrer un mouvement et de provoquer le déplacement de tout ou partie du système. Le corps humain est ainsi ramené à un corps quelconque ou à un système de corps ${ }^{4}$.

On sait que la mécanique biologique est devenue la manière privilégiée de se représenter le corps sportif, c'est-à-dire un corps circonscrit par l'abstraction du regard scientifique comme structure morphologique, osseuse, organique et musculaire. Cette

4. A. LEROY et J. VIVES, Pédagogie sportive et athlétisme, Paris, Éditions Bourrelier, 1949, p. 65 . 
représentation du corps a des visées bien déterminées : par exemple, solliciter et améliorer les grandes fonctions corporelles (circulation, respiration, élimination), pallier les insuffisances morphologiques et fonctionnelles, acquérir plus de souplesse articulatoire et un meilleur rendement musculaire. Cette conception est reprise et accentuée par $A$. Lapierre :

L'être humain, du point de vue mécanique, peut être envisagé sous la forme d'un ensemble complexe de leviers osseux, reliés par des charnières articulaires variées et mus par la contraction musculaire. Ce complexe mécanique obéit aux lois communes des leviers, il repose sur le sol par des appuis variables et est soumis à l'action de la pesanteur et aux lois de l'équilibre. Les mouvements sont eux-mêmes des actions mécaniques déterminées 5 .

Enfin, on trouve dans un manuel de physiologie humaine fréquemment utilisé par nos étudiants universitaires, l'affirmation suivante: "Man, then, is a machine, an enormously complex machine, but a machine, nevertheless" 6 .

En réalité, toutes ces opérations du corps humain débordent leur stricte dimension physiologique parce qu'elles sont humaines, et dans la mesure où elles sont ordonnées à l'apprentissage de la santé, à l'accoutumance à l'effort, au dépassement de soi-même et à l'ajustement du corps au monde extérieur ; elles reçoivent ainsi une résonance psychologique, morale et sociale. En fait, cet ensemble d'interventions sur le corps connote des normes essentiellement culturelles, plus particulièrement techniques. En effet, ce corps biologique, à la fois anatomique et physiologique, renvoie à un corps-instrument au service d'une recherche exacerbée de rendement. Et lorsque la technique sportive prend en charge le corps biologique, c'est pour le construire d'abord au moyen d'un entraînement physique rationnel, axé sur des objectifs de rendement musculaire, sur la pratique d'un travail généralisé de tout le corps, sur le rôle capital de la coordination motrice générale, ainsi que du développement et de la maîtrise de certains réflexes. Elle fait ainsi apparaître un corps instrumentalisé de part en part.

5. A. LAPIERRE, La rééducation physique, T.I., Paris, J.B. Ballière et Fils éditeurs, 1968, p. 79.

6. A.J. VANDER, J.H. SHERMAN, D.S. LUCIANO, Human Physiology. The mechanisms of body function, Introduction, University of Michigan, 1975, p. 1. 
Cependant, l'obtention d'un tel rendement n'est pas le fruit du hasard. Au contraire, la nécessité d'une efficience maximale du corps implique une individualité minimale. En d'autres termes, le système sportif fonctionne d'autant plus efficacement et le corps sportif est d'autant plus productif que, d'une façon générale, les individus sont réduits à des unités purement quantifiables, et, en particulier, leur corps est fractionné en blocs d'organes, de muscles, d'énergies, de gestes fonctionnels. Cette parcellisation permet de mieux envahir le corps, désormais éclaté comme un terrain ouvert, favorisant ainsi sa meilleure technicisation et permettant de prévenir le geste maladroit ou inutile : le geste construit techniquement acquiert une promptitude, une précision et une efficacité mécaniques; il est soustrait à la lenteur et à l'incertitude de la réflexion. En effet, et c'est là une autre manière de façonner le corps, pour qu'un geste atteigne une certaine infaillibilité, il doit échapper le plus possible à l'emprise de la subjectivité. On se convainc que l'individu, livré à sa seule volonté ou à sa seule intelligence, est limité dans son action ; dès lors, sa pleine efficacité devra passer par l'utilisation de techniques de motivation et de concentration, ainsi que par l'autorité de l'entraîneur-éducateur professionnel, afin de plier cette volonté et cette intelligence aux exigences du système sportif et de rendre plus obéissant ce corps instrumentalisé. Il est particulièrement remarquable que dans le système sportif, l'entraîneur incarne l'unité fonctionnelle : n'est-il pas au cœur de la pratique sportive ! C'est lui qui médiatise et unit dialectiquement l'administration des choses et le gouvernement des individus : par son savoir technique, il incarne et transmet l'autorité de la technique ; il véhicule aussi les valeurs de discipline, d'ordre, de pouvoir, de maîtrise, de sécurité, de dépassement de soi, d'esprit d'équipe, toutes propres à l'idéologie productiviste : en ce sens, il est un idéologue inavoué.

Ainsi, grâce à une technicisation sportive prégante du corps, on assiste à une exploitation systématique et rationnelle des aptitudes psychomotrices de chaque individu, suivant le schéma du processus de production industrialiste : compétition, rendement, mesure, record, avec ses exigences de division et de spécialisation des tâches. La fabrication du corps passe donc par le contrôle technique de l'énergie physique de l'individu dans un 
temps et un espace planifiés et programmés ; elle s'associe aussi les techniques d'apprentissage de la résistance à la fatigue, de l'accoutumance à l'effort, de la concentration et du dépassement de soi. Cette technicisation des énergies vitales de l'individu n'a pas d'autre but que la recherche de l'efficacité, une efficacité bien ambiguë cependant puisque derrière des finalités humanistes de maîtrise du corps, d'amélioration des qualités psychologiques et des rapports aux autres, s'impose la finalité du productivisme. C'est ainsi que les gestes et les mouvements du corps, leur perception et leur rationalisation, tendent à être unifiés et intégrés dans une même finalité du rendement technique ; il n'y a pas ou peu de place pour l'improvisation, pour la spontanéité, pour la liberté physique et ludique. Le corps devient alors une mosaiqque d'énergies fonctionnelles soumises à une totalité de techniques additionnées et concordantes ; il est réduit à une puissance étrangère à l'individu ; il est l'objectivation de sa puissance abstraite de produire des efforts réglés et efficaces.

Certes, ce processus de technicisation du corps, et partant de tout l'individu, atteint d'abord et surtout une élite sportive. Cependant, il ne s'y confine pas. L'immense domaine des activités physiques et sportives de loisir destinées à la masse, celles qui sont promues et développées pour des fins éducatives, ainsi que l'ensemble des activités humaines liées à un enjeu quelconque n'échappent pas à l'influence de la technique sportive : celle-ci pénètre plus ou moins profondément ces mondes par la force d'un conditionnement psychomoteur et sociologique, grâce, d'une part, à la propagande officielle qui consacre la réussite de l'athlète et le prestige du champion national comme fleurons de l'intervention de la technique de pointe, et, d'autre part, grâce à la publicité et à l'organisation commerciale des activités physiques et des loisirs, promettant ainsi une restauration efficace, parce que cautionnée par la technique, des énergies vitales de l'individu en vue d'assurer son équilibre psycho-physique et sa meilleure intégration dans le système social.

En somme, par une objectivation, une standardisation et une atomisation des mouvements du corps, dans l'optique d'un modèle techniciste d'un corps-instrument, le sport moderne est devenu la science expérimentale du rendement corporel, selon le principe de fonctionnement de la production industrialiste et 
de la rationalisation par le calcul systématique. Par ailleurs, que cette technicisation du corps séduise l'homme moderne et plus particulièrement le sportif, cela s'explique par le fait que la technique est devenue l'instrument privilégié du désir : en effet, par la technique, l'homme cherche à imposer sa puissance, à se rendre maître des cadres de son existence et de l'humain luimême, à vivre une sorte d'extase qui puisse l'arracher à lui-même et lui permettre de se faire démiurge. D'où un glissement subreptice vers une autre modélisation du corps qui renvoie à ses énergies libidinales et agonales.

\section{Le sport comme systématisation de l'instinct agonal}

Cette modélisation sportive du corps n'est pas exclusive au plan anatomo-physiologique. Elle entraîne et structure aussi toutes les forces agonales d'un corps chargé d'agressivité. Pour mieux saisir le processus de systématisation des pulsions agonales de l'individu humain par le sport, nous croyons pertinent de rappeler ce qui, à notre avis, caractérise l'actuelle civilisation occidentale.

Tout indique que nous vivons à l'époque d'une société acquisitive, c'est-à-dire une société fondée sur la propriété privée ${ }^{7}$, le profit et la puissance. Dès lors, acquérir, posséder et réaliser des gains sont devenus graduellement des besoins impératifs et, à la limite, des droits inaliénables de l'individu humain vivant en sociétés techno-industrielles axées sur la production et la consommation systématiques. Or, dans ce mode acquisitif d'existence, chacun est comme mis en demeure de tirer sa satisfaction et son bonheur non seulement de l'affirmation de soi, mais aussi et surtout de sa supériorité sur les autres et de sa propre puissance par voie compétitive. Suivant la logique de la propriété privée, c'est-à-dire des diverses formes de possession ou d'incorporation physique, symbólique et magique, et du profit qui en découle, chaque individu est de plus en plus profondément imprégné de ce mode d'existence compétitif, presque toujours à son insu, alors même que les moyens matériels d'y accéder peuvent être

7. La réalisation de la vie humaine a besoin de la propriété privée. Le plus virulent critique de la propriété privée, Karl Marx, n'a jamais contesté ce fait : il y a une nécessaire propriété privée, au sens d'appropriation des biens légitimée par le travail ; ce que Marx fustigea, c'est la propriété des moyens de production non acquise par le travail. 
pratiquement inexistants pour un bon nombre de consommateurs. Mais qu'à cela ne tienne, puisque, comme nous venons de le suggérer, l'acte consommatoire n'est pas qu'un processus d'appropriation et d'absorption matérielles de biens et de services visant à satisfaire des besoins ; il est davantage "une activité de manipulation systématique de signes ${ }^{8}$. Autrement dit, par delà la matérialité des objets, c'est leur fonctionnalité qui est possédée et consommée. Or, cette façon d'entrer en rapport avec les choses peut difficilement être refusée à quiconque, dans la mesure où la dynamique de son acte d'appropriation et de consommation est celle d'un acte investi par le désir et l'imaginaire. En ce sens, nul n'échappe au mode avoir d'existence et, par conséquent, à son principe énergétique qu'est la compétition. En effet, dans nos sociétés techno-industrielles, axées sur la production et la consommation systématiques, dominées par le principe du rendement, il est considéré comme normal et important d'avoir l'esprit de compétition pour exister, assurer sa carrière, obtenir du succès, du prestige et du statut. Dès lors, la compétitivité est posée comme structure structurante des rapports humains ; d'ailleurs, la complexité et la richesse même de son contenu nous invitent à la percevoir ainsi.

C'est du moins ce que nous indiquent les nombreuses analyses de la structure de la compétition, qui enseignent que la compétitivité peut être fonction de la conjugaison d'une triple série de facteurs : les caractéristiques génétiques de l'individu, le cadre environnemental dans lequel il vit et les aspects situationnels de son environnement actuel ${ }^{9}$. Dans cette perspective, un comportement de compétition est initialement engendré par des pulsions primaires qui s'intègrent à la structure de la personnalité, elle-même travaillée par l'environnement socio-culturel dynamisé par des pulsions secondaires de prestige, d'admiration, de statut et de gain. Phénomène et comportement à la fois biologiques, psychologiques et sociaux, marqués par un rapport de lutte entre deux ou plusieurs individus, sous l'effet d'un

8. Jean BAUDRILLARD, Le système des objets, Paris, Gallimard, 1968, p. 233.

9. Parmi les nombreuses études du comportement compétitif nous signalons les suivantes : Konrad LORENZ, L'agression, une bistoire naturelle du mal, 1969 : A. MONTAGU, The bumanization of man, 1960 ; R. ARDREY, The territorial imperative, 1966 ; J. DOLLARD et N.E. MILLER, Frustration and agression, 1939 ; C.L. HULL, Principles of behaviour, 1943. 
renforcement lié à la performance relative de chacun pour atteindre un objectif commun ou obtenir un résultat donné, la compétition constitue le nerf vital du mode avoir d'existence en général, et, plus particulièrement, de tout rapport humain marqué au coin d'un enjeu. On comprend que, dans un contexte sociétal acquisitif, l'individu soit contraint de faire ses preuves et de s'imposer aux autres s'il veut réussir, qu'il lui arrive même de souffrir d'un besoin névrotique de comparaison quantitative et qualitative pour savoir ce qu'il est et ce qu'il vaut.

Il fallait évoquer cette caractéristique déterminante de notre civilisation occidentale pour éclairer la dimension compétitive du sport, puisqu'il en porte la marque profondément. À notre avis, de toutes les formes d'activités humaines parvenues au statut d'institution et de système, le sport moderne est devenu celle qui présente actuellement le plus de force et de cohésion des pulsions humaines de compétitivité : n'est-il pas la théorie et la pratique des pulsions agonales de l'individu humain?

D'une part, le sport moderne constitue la systématisation la plus rigoureuse et la plus efficace de ce qu'il y a d'agressivité, de performativité et de lutte pour l'accession à la supériorité et au prestige suprême chez l'individu humain. En effet, si la compétition peut être définie comme une lutte entre individus ayant des prétentions rivales sur le même enjeu, c'est dans la mesure où elle contribue à mobiliser des énergies vitales spécifiques, c'est-à-dire des énergies agressives. L'agressivité peut être représentée comme un complexe de pulsions instinctivo-volontaires qui s'expriment dans des situations de lutte, c'est-à-dire des situations exigeant un déploiement constant d'efforts d'affrontement ou de confrontation. Nous donnons au terme "lutte" son sens le plus général, c'est-à-dire celui d'une tension énergétique fondamentale propre à toute existence concrète. Chez l'humain, cette tension énergétique prend les formes les plus diverses, tant physiques que psychologiques ; ainsi, l'individu humain n'existe que dans la mesure où il se maintient dans l'existence par ses forces physico-biologiques et par le dynamisme de ses choix volontaires. Dans cette optique, l'agressivité peut être perçue comme un moyen de franchir ou encore de supprimer les obstacles qui s'opposent à la satisfaction du besoin humain d'exister. 
La question d'origine exogène ou endogène de l'agressivité n'intéresse pas notre étude. Il importe, par ailleurs, de signaler que le concept d'agressivité oscille entre l'esprit d'initiative et de recherche, d'aventure et de victoire sur les difficultés opposées par le milieu ou la vie, et la volonté sadique de détruire : c'est Éric FROMM qui nous a appris à distinguer une agressivité défensive que l'homme partage avec l'animal et qui assure sa survie, et une agressivité maligne qui incite l'homme à tuer sans but social ni nécessité biologique et qui est spécifiquement humaine ${ }^{10}$. En principe, il faut exclure du sport la destructivité, cette forme maligne de l'agressivité, pour n'y reconnaître que l'agressivité qui fait référence à un comportement axé sur la récompense (réussite, prestige, victoire), dont le but est d'atteindre un certain objectif et dans lequel le dommage causé à autrui n'intervient que parce que c'est le moyen le plus efficace de parvenir à l'objectif recherché. La pratique n'est pas toujours aussi condescendante ! À tout le moins, le sport fait essentiellement appel à ce complexe de pulsions instinctivo-volontaires, à une agressivité positive et instrumentale, nécessaire à la performance du sportif : c'est une agressivité que des règles codifiées viennent canaliser et rendre légitime. Dans le sport, on lutte physiquement et volontairement, tant contre soi-même et sa nature, sa pesanteur matérielle et son hésitation ou sa peur, que contre son adversaire et la volonté d'autrui ; mais cette lutte, prix de la victoire, est codifiée, limitée, consentie. C'est en cela d'ailleurs que l'agonistique sportive sert de référent aux autres activités humaines.

D'autre part, et plus fondamentalement encore, le sport moderne s'est graduellement imposé comme agent de formation de l'individu humain pris dans sa totalité. Le processus de sportivisation s'est approprié l'individu total, tant dans son corps et son esprit que dans ses activités singulières et collectives. Toutefois, ce procès de la sportivisation est d'abord et avant tout celui du corps ; s'il atteint la conscience de l'individu, c'est parce qu'il traverse d'abord un corps-machine, un corps foyer d'agressivité, de désirs et de fantasmes. Ainsi que nous y avons fait allusion plus haut, deux raisons expliquent cet intérêt pour le

10. Éric FROMM, La passion de détruire, Paris, Éditions Robert Laffont, 1975. 
corps. D'une part, le corps aujourd'hui, au lieu de constituer une instance vivante et contradictoire, a tout simplement pris le relais de l'âme comme instance mythique et comme objet de salut, héritant ainsi de sa fonction idéologique, devenant par là même le support privilégié de l'objectivation et d'un système productiviste évolué. D'autre part, le corps, structure anatomophysiologique, est aussi représenté comme un système d'énergies agonales et libidinales : tel est, nous l'avons déjà souligné, l'enseignement de la psychanalyse. Or, pour devenir une force productive efficace, cette mosaïque d'énergies physiques, agonales et libidinales doit être conjurée, domptée et réappropriée selon une contrainte d'instrumentalité indexée sur les normes d'une société de production et de consommation systématiques : corps/ fétiche qu'il faut investir narcissiquement, non pas pour le connaître en profondeur et pour en jouir, mais pour le constituer comme objet plus lisse et plus fonctionnel, c'est-à-dire comme corps/capital. Or, dans la mesure où le sport moderne est une mobilisation rationalisée des divers processus énergétiques du corps, il se présente comme l'organisation la plus rigoureuse et la plus efficace pour dompter et gérer ces énergies, contribuant ainsi à aménager le corps comme un patrimoine et à le manipuler comme un des multiples signifiants de statut social ; la sportivisation du corps devient la manière privilégiée de censurer le corps dans sa vérité subversive de l'agressivité et du désir. Dès lors, le sport moderne n'est pas vraiment la négation du corps ; au contraire, il est sa réhabilitation, mais une réhabilitation orchestrée moins selon les finalités autonomes du sujet que selon une contrainte d'instrumentalité et un principe normatif de rentabilité. C'est ce corps ainsi sportivisé, donc objectivé, qui tend à devenir le grand médiateur de notre civilisation.

Si donc le sport moderne fonctionne comme mobilisateur systématique des énergies agressives et libidinales de l'individu, s'il est une rencontre agonistique de soi avec soi et de soi avec l'autre, tant physique que volontaire, on peut difficilement lui attribuer une simple fonction de reflet et de reproduction du système social global. Il en fait partie intégrante, certes ; mais en tant que partie, ou mieux en tant que sous-système, il exerce aussi et surtout une fonction productrice et constructive massive ; il agit comme modèle diffus de rationalisation des énergies 
corporelles et psychiques, partout où celles-ci sont mises à contribution pour l'obtention d'une performance. C'est pourquoi, après avoir été généré par le ssytème social global, le sport en est arrivé à puiser dans ce même système les éléments et la logique nécessaires à sa propre systématisation, au point d'en devenir un référent. Ainsi, le sport moderne est devenu le fer de lance du système social, en imprimant à toute la conscience de l'homme contemporain, sa structure, ses valeurs et son idéologie : quel que soit le secteur, partout où la comparaison quantitative, la performance mesurée, le rendement calculé, l'agressivité, les règles et les stratégies de l'action agissent, ce sont en tant que valeurs marquées de sportivisation. Le sport devient la nouvelle logique du comportement agressif, performatif et compétitif. Il s'impose comme le schéma directeur ou le modèle formel de tous les cas possibles de compétition à instaurer entre les humains.

Par ailleurs, et suivant l'optique que nous tentons d'expliciter, s'il est vrai que la compétition constitue un caractère fondamental et le dynamisme de l'activité sportive, elle ne la définit pas d'une façon spécifique. Il y a une polysémie du sport qui nous empêche de le réduire à l'une ou l'autre de ses caractéristiques, que ce soit le jeu organisé, l'effort physique intense, l'enjeu, les règles, la compétition, etc.. À notre avis, la compétition est d'abord et avant tout l'essence des rapports humains, dans la mesure où ceux-ci conjuguent des éléments de coopération et d'opposition, un avec et un contre ${ }^{11}$. C'est ce fond originel que l'activité sportive prend en charge ; ces forces agonistiques, elle les condense, pour ainsi dire, dans l'univers des corps et des volontés, et les rationalise dans son système de règles pour les rendre plus efficaces et éthiquement acceptables. En somme, l'ordre sportif s'impose à l'ordre social parce qu'il réalise avec plus d'efficacité et de cohésion les exigences et les objectifs même du système social global, en travaillant avec succès sur tous les registres du symbole de la réalité socio-politique qu'est le corps humain, plus spécifiquement en imposant un nouveau corps sain et agressif, dompté et obéissant, efficace et exemplaire : le corps sportif.

11. Michel BOUET, Signification du sport, Paris, Éditions universitaires, 1968, p. 52. 


\section{Le sport comme hiérarchisation des corps}

Un autre trait marquant contribue à poser le sport moderne comme repère central : c'est le fait qu'il évolue dans un esprit profondément élitiste. C'était là d'ailleurs un des objectifs majeurs du restaurateur des jeux olympiques modernes, le baron Pierre de Coubertin, pour qui un peuple fort ne peut naître sans une population aguerrie par les exercices physiques et les sports : "C'est toujours l'élite que l'on a en vue, car une phalange supérieure et peu nombreuse rend infiniment plus que la médiocrité très répandue ${ }^{12}$. Le sport moderne s'est ainsi élaboré selon une structure hiérarchisée, prenant la forme d'une pyramide, où les individus sont évalués en fonction de leurs aptitudes performatives mesurées.

Le système sportif n'a certes pas inventé cette façon de catégorier les individus et leurs rôles. Tout corps social repose sur la hiérarchie des individus en fonction de leurs rôles, de leur statut, de leur pouvoir et de leurs aptitudes. Les sociétés les plus démocratiques ou les plus égalitaires n'échappent pas à ce processus. La raison fondamentale réside sans doute dans le fait même des inegalités naturelles et produites entre les individus. Or, cette discrimination naturelle et sociale crée normalement des différenciations fonctionnelles, notamment des fonctions d'autorité et d'exécution. En outre, ce processus de hiérarchisation s'établit en raison de l'importance que les individus attachent aux distinctions de rang et de pouvoir ; il est particulièrement inscrit dans les symboles d'une société : ainsi, tout objet approprié peut devenir un signe indiquant le statut qu'on occupe, le pouvoir qu'on exerce, le prestige dont on jouit. En somme, il n'y a pas de système social sans différences, c'est-à-dire sans fonctions et sans mise en relation des éléments différenciés et fonctionnels qui le constituent. La fonctionnalité des éléments du système est particulièrement importante pour que cette mise en relation s'exerce de manière efficace et assure un ordre sys-

12. Pierre de COUBERTIN, " L'éducation anglaise ", in La réforme scolaire, 1887, p. 649. Dans l'esprit de Coubertin, l'olympisme suppose une aristocratie des athlètes qui composent une élite démocratiquement basée sur l'égalité des chances au départ : «Pour que cent se livrent à la culture physique, écrit-il, il faut que cinquante fassent du sport ; pour que cinquante fassent du sport, il faut que vingt se spécialisent; pour que vingt se spécialisent, il faut que cinq soient capables de prouesses étonnantes. Impossible de sortir de là. Tout se tient et s'enchaîne. " (Pédagogie sportive, Vrin, 1972, p. 55). 
témique ; c'est pourquoi les éléments ne sauraient être traités comme autonomes, mais plutôt d'une manière ordonnée et hiérarchisée : l'existence même du système et l'ordre proprement dit en dépendent.

L'instauration d'une hiérarchie dans le sport a pris naissance dans le système social global ; elle est devenue par la suite un modèle. En effet, d'une part, le sport moderne consacre l'échelonnement des individus dans une pyramide sociale en fonction de leurs aptitudes et de leurs performances mesurées à partir des critères du système sportif : il doit y avoir un champion et le meilleur doit être manifesté. Cette hiérarchisation, qui procède par classement et sélection à l'intérieur de règlements bien définis à l'avance, est particulièrement importante. Elle permet d'identifier le vainqueur, ce symbole d'efficacité et d'excellence, qui exercera sur la foule des consommateurs sportifs une influence magique et mystificatrice. En outre, elle est nécessaire si l'on veut assurer le contrôle des énergies agonales qui alimentent le système sportif et l'ordre des rapports entre les individus concernés : elle met chacun à sa place, au nom même de l'efficacité du système. D'autre part, la hiérarchie sportive apparaît comme un modèle d'ordre et d'efficacité : elle en est le positivisme. Dans le sport, les hiérarchies sont précises, rigoureuses, évidentes ; plus qu'ailleurs, la raison satisfait son besoin d'ordre et d'efficacité, sans doute parce qu'elle peut, mieux qu'ailleurs, machiner le corps transformé en outil polyvalent et ainsi chercher à le neutraliser en tant que substance désirante et agressive. Suivant cette logique de la raison sportive, le sportif doit prouver ses aptitudes en passant des examens de toute sorte ; il est alors soigneusement classé par sa valeur sportive, mais une valeur fonctionnelle puisque entièrement axée sur le système. Ainsi soumis au moule formel des activités proposées, souvent imposées, le sportif peut gravir les échelons de la hiérarchie jusqu'à faire partie de cette aristocratie auréolée de prestige qu'est le groupe de champions.

Le sport moderne devient alors la consécration objective de l'effort constant et patient, des valeurs physiques et psychologiques, de la soumission à une logique systématique ; le système sportif apparaît comme l'art et la science de créer une nouvelle société d'individus : il y a ceux qui incarnent la raison sportive 
et les fonctions d'organisation et de pouvoir qu'elle assure ; il y a ceux qui mettent leur volonté sportive au service de cette raison pour en exécuter les ordres; il y a ceux qui intègrent psychiquement les idées directrices de cette raison et se laissent séduire par les exploits de cette volonté ; à l'horizon de cette triade, il y a ce personnage indubitable, ultime création du système sportif: le champion. Celui-ci est particulièrement important puisqu'il symbolise l'efficacité et l'excellence ; il en est la preuve incontestable ; il est un idéal concret de courage, de ténacité et de puissance. En outre, le champion fascine dans la mesure où, produit d'un long processus d'entraînement forcené et spécialisé, il est le résultat d'une quantophrénie, pour reprendre le mot de $\mathrm{P}$. Sorokin, c'est-à-dire d'un besoin névrotique d'objectivité mesurée ou du prestige du quantitatif ; en effet, ce qui est de l'ordre du quantitatif se saisit immédiatement, facilement, puisqu'il s'agit de quelque chose qui est en dehors de toute subjectivité et qui vaut universellement. Dès lors, le champion est l'aboutissement normal de la magie du chiffre, lui-même symbole achevé des œuvres d'un esprit de géométrie.

En ce sens, le système sportif fait plus que refléter la hiérarchie sociale et le processus d'objectivation du travail qui en résulte. Il est un modèle de hiérarchisation efficace et d'organisation rigoureuse des activités humaines vouées à la performance par l'utilisation systématique de la mesure et du résultat : l'individu vaut ce que valent sa performance et son résultat, en référence à un temps rationnel, mécanique et linéaire.

\section{Le sport comme repère de mythes}

La modélisation sportive du corps humain comme structure anatomo-physiologique et comme matière désirante et agressive en un corps transparent et dressé de part en part, camoufle cependant une dimension complexe et confuse : celle d'un corps comme «symbolique générale du monde ${ }^{13}$. Le corps humain, tel que nous avons tenté de le circonscrire, récapitule en toutes ses parties les significations des choses et des êtres qu'il perçoit

13. Maurice MERLEAU-PONTY, Phénoménologie de la perception, Paris, Gallimard, 1945, p. 274 
et sur lesquels il agit ; plus profondément encore, il est à l'origine de tous les autres symboles, leur référent permanent. C'est pourquoi on a pu parler d'un double symbolisme corporel : l'un qui est tourné vers l'expérience libidinale du corps, l'autre qui renvoie à la situation sociale qui lui donne sa signification. C'est pourquoi aussi on ne peut dire et atteindre le corps humain qu'à travers la diversité des discours symboliques historiques, c'està-dire à travers une multitude de mythes forgés par les cultures ${ }^{14}$.

Or, cette mythologie diversifiée a trouvé dans le sport moderne une expression nouvelle, dont le corps sportif constitue la dernière forme. Comment le sport moderne est-il devenu un système solidement structuré et cohérent de mythes et, partant, une source privilégiée et l'exutoire de l'imaginaire individuel et collectif ? La question est vaste et complexe; elle appelle une réponse que nous ne pouvons pas établir ici. Nous tenterons plutôt d'expliciter en quoi le système sportif se renforce par une production constante de mythes. Nous ne chercherons pas cependant à répertorier toute cette production; l'entreprise nous semble d'ailleurs impossible dans la mesure où cette production est l'acte incessant d'une conscience imageante sans limites assignables. Notre contribution vise à prolonger les analyses déjà proposées de quelques-uns de ces mythes sportifs ${ }^{15}$.

D'une part, les thèmes essentiels de la mythologie du sport (neutralité du sport, sa pérennité, son idéologie du progrès et du record, la participation sportive de masse, le champion, le héros, le spectacle sportif, etc.) expriment, reflètent et sauvegardent la logique et les propriétés du système sportif où les contradictions, les déchirures et les antagonismes nécessitent une réponse imaginaire. Là comme ailleurs, de nombreuses pratiques et opinions sont soumises à la souveraineté du mythe. À tel point que de nombreuses pratiques sportives sont connues de

14. Michel BERNARD, Le corps, Paris, Éditions universitaires, 1972, p. 129-237 ; W. PASINI, Éros et changement, Paris, Payot, 1981, p. 33-36.

15. Quelques auteurs se sont intéressés à la mythologie sportive, par exemple : J.M. BROHM. Le mythe olympique, Paris, C. Bourgeois éditeur, 1981 ; en collaboration, Des mythes sportifs, coll. Les Dossiers Beaux-Jeux, Montréal, Bellarmin, 1978. C'est ainsi qu'on a fait état des mythes de l'origine primitive du sport, de la pérennité du sport à travers les âges, de la neutralité du sport, de l'idéologie du progrès et du record, de l'information sportive, de la gratuité, de la participation sportive de masse, du champion du spectacle sportif, etc.. 
l'opinion publique plus par l'image que le mythe en reflète que par une étude objective et directe des structures du sport et des rapports sportifs. Le mythe, c'est-à-dire ce discours de l'esprit imaginant et, à la limite, cette façon illusoire d'appréhender une réalité historique et de la vivre, devient alors la source d'un savoir sportif, une sorte de modèle auquel les producteurs et les consommateurs sportifs se réferent pour maintenir l'ordre des choses et pour nourrir leur monde imaginaire. Cette mythification comporte une part d'idéologie, dans la mesure où elle exerce une fonction justificatrice dont a besoin la mentalité collective et qu'utilisent bien les gardiens de l'ordre et de l'autorité. Sous cet aspect, les mythes sportifs contribuent à camoufler derrière leur écran imaginaire les réalités historiques concrètes du système, la prégnance du politique sur les activités sportives et sur les activités humaines en général, les conflits qui tissent les rapports humains de cet ordre. Davantage, ils trahissent ces faits en les amplifiant et en les déformant pour en faire des normes de jugement et de conduite collectivement recevables. Telle est d'ailleurs la double nature du mythe. Ce faisant, la mythologie sportive produit une adhésion acritique des individus à ce qu'elle représente comme l'inverse du politique, c'est-à-dire le sport. Cette mythologie est donc porteuse d'une insignifiance politique des choses et des actions humaines. Telle nous semble être la portée des mythes de la trève des jeux olympiques, de la pureté du sport et de l'information sportive : mythes sécurisants de la réconciliation, de la suppression de la lutte des classes sociales, de l'abolition des conflits raciaux et nationaux, de la normalisation de l'événement, du sport neutre, innocent et vierge, qui aurait été perverti par ses mauvaises utilisations socio-politiques.

D'autre part, le sport moderne apparaît comme la plus habile confirmation dans la croyance au progrès, mais à un progrès essentiellement quantifiable et linéaire, constant et sans limites assignables. L'idée de progrès est devenue une croyance fondamentale du monde occidental, alors même que sa naissance et sa première élaboration ne datent que du XVIII ième siècle. D'abord liée à l'idée d'une révolution par la raison et la science pour plus de liberté, d'égalité et de justice, la notion de progrès a été pensée par la suite sous les catégories de puissance et de pouvoir d'une raison techno-industrielle et économique. Nous 
obtenons, en conséquence, une version productiviste et unidimensionnelle du progrès, puisqu'elle soumet les objectifs humains au calcul rationnel dont l'instrument le plus fiable est le chiffre.

Or, cette suprématie du «plus» a été réappropriée et amplifiée par le sport moderne. Dans le sport moderne, le progrès psycho-physique humain est objectivé par celui des performances sportives, elles-mêmes mesurées par l'extension de la pratique compétitive et du nombre de pratiquants dont l'étalon suprême est le record : c'est un monde soumis à la fascination du chiffre. Or, le chiffre fascine parce qu'il nous campe d'emblée dans l'ordre de la représentation, c'est-à-dire dans le monde même de la raison imaginante. En tant que représentation, le chiffre avise un objet réel concret, mais la matière du chiffre est totalement indifférente à l'objet signifié : à la limite, il importe peu que telle distance ait été franchie par tel athlète, puisque celui-ci est devenu une sorte de fantôme quantitatif, en incarnant abstraitement un chiffre, un record ; il est devenu une image mathématique derrière laquelle se dissimule un individu vivant avec des qualités vivantes sans signifiance. Ce qui fascine dans le chiffre, c'est son aspect immatériel, symbolique, manipulable : le chiffre est le symbole de la puissance rationnelle sur les corps.

Ce caractère fantasmagorique du chiffre pose au corps humain une question centrale : celle de la limite biologique de l'individu humain. Il est vrai que l'homme est le seul être de la nature à s'imposer une progression de ses forces corporelles, à se poser la question de l'infini et de l'absolu de son corps. Une loi de la valeur symbolique l'habite et lui dicte que l'essentiel est toujours au-delà de l'indispensable : l'individu humain ne vit et ne pense que pour ce qui n'est pas encore; l'infini est indispensable au fini, il en est son dynamisme universel. Il existe cependant une disproportion, une dichotomie, entre l'objet du désir et le besoin d'infini, si bien que le désir d'infinitude peut constituer une sorte de point aveugle, dans la mesure où il occulte une prise de consciençe réaliste des limites du corps.

Or, le sport moderne reprend à son compte ce désir d'infinitude et soumet par là même tout le corps humain à une progression infinie. Par le biais du sport, non seulement l'homme exprime sa puissance sur la nature et sa domination d'autrui, 
mais il affirme aussi l'idée du dépassement de soi et l'idée d'immortalité : grâce au dépassement qui appelle de toutes ses forces un corps investi par le désir et travaillé par la technique, ellemême instrument du désir, l'individu humain est séduit par l'idée d'immortalité ${ }^{16}$. En réalité, le sport reste le vécu d'une dialectique de l'infinitude et de la finitude humaines. Davantage, il est une dramatisation révélée par une violence codifiée et consentie, par l'incertitude du résultat et surtout par le jeu symbolique de la mort. Mais cette dramatisation est faussée par une raison sportive productiviste qui l'institue en dépositaire d'une célébration de la performance quantitative dans le rituel compétitif. Le sport est alors posé comme ascension fantastique de l'homme à la condition de surhomme biologique ou d'homme bionique. Tel est le mythe du progrès sportif.

$\mathrm{Ce}$ mythe en génère un autre : celui du champion. Celuici est la conséquence logique de l'affirmation du dépassement de soi et du désir d'immortalité : grâce à l'idée d'immortalité créée par une conscience imageante et inscrite par une technique fantastique dans le corps, l'individu sportif dépasse le commun des mortels et se place sur la voie de l'élite, du champion, du héros. Le champion devient un mythe dans la mesure où il est constitué en modèle de rationalité sportive et en schéma d'action possible. Il résume et réalise une triple adéquation de l'homme avec lui-même : d'une part, il est perçu comme celui qui a parfaitement réussi à mâtriser la mécanique de son corps et la force anarchique de ses impulsions et à en connaître les possibilités ; d'autre part, il révèle la conformité possible entre les objectifs visés et les moyens de les atteindre; enfin, il crée son propre modèle puisqu'il n'imite rien et ne se projette sur personne : il est identique à soi. Cette sorte de demi-dieu, création d'une pensée imaginante qui, tout en prenant appui sur des faits, les amplifie et les plie à ses exigences, contribuera à colmater les conflits psychologiques ou socio-culturels de l'individu humain ${ }^{17}$. En ce sens, on peut dire que l'histoire sportive devient la fétichisation idéaliste des champions.

16. Le vocabulaire sportif traduit bien cette idée et l'activité sportive porte les stigmates d'une déification de l'homme.

17. La production du champion est une sorte d'acte magique - celui de la technique une incantation destinée à faire apparaître l'objet qu'on désire, de façon qu'on puisse en prendre possession; il y a cependant dans cet acte toujours quelque chose d'impérieux et d'enfantin, un refus de tenir compte de la réalité conflictuelle. 
Par ailleurs, le sport ne se présente pas seulement comme action : il est aussi spectacle. Cette dimension du sport constitue, à notre avis, une des sources privilégiées et l'exutoire par excellence de l'imaginaire individuel et collectif. Qu'est-ce qui fait que le sport est devenu objet de spectacle, qu'est-ce qui constitue le dynamisme du spectacle sportif ?

Une certaine critique du sport moderne, marxisante le plus souvent, s'emploie à en dévoiler les mécanismes idéologiques. C'est ainsi que le spectacle sportif est interprété comme la consécration visible du sport qui se vend, qu'on loge le dynamisme du spectacle sportif dans la valeur marchande, que celui-ci est réduit au statut de consommation culturelle qui correspond strictement aux besoins de la production culturelle de masse du système socio-économique. Cette critique dénonciatrice va encore plus loin : elle soutient, par exemple, que le spectacle sportif est devenu un facteur de stabilisation de l'ordre social existant et de l'enrégimentement des foules, le rituel obsessionnel de masse d'une société répressive, une sorte d'opium du peuple au double sens du terme : « d'une part, obscurcissement des facultés critiques (évasion, fuite, extase) et d'autre part, compensation, substitution pour les malheurs réels ${ }^{18}$.

Ces analyses ne manquent pas de pertinence. Il est possible cependant d'élargir leur grille et d'élaborer une interprétation du spectacle sportif axée sur la signification de ces deux instances : le spectacle lui-même et le regard. Nous proposons, pour l'instant, une réflexion embryonnaire sur l'une et l'autre de ces réalités ${ }^{19}$.

Le spectacle est de l'ordre de la représentation, non de l'action proprement dite : il est une composition de la conscience perceptive et de la conscience imageante. En effet, le spectacle n'est tel que parce que l'événement qui se déroule dans un espacetemps déterminé est vu. C'est l'acte perceptif et imageant de la conscience qui transforme un événement en spectacle. Cepen-

18. Pierre LAGUILlAUMIE, "Pour une critique fondamentale du sport ", in Sport, culture et répression, Paris, Maspero, 1972, p. 54. Les analyses virulentes de J.M. BROHM vont dans le même sens.

19. Une phénoménologie du spectacle sportif et du sport comme objet de regard reste à faire L'étude de Guy DEBORD, La société du spectacle, Paris, Buchet/Chastel, 1967 et les analyses sartriennes du regard nous paraissent constituer des références pertinentes. 
dant, percevoir et imaginer ne sont pas deux types de consciences identiques par lesquelles un même objet peut nous être donné. Dans la perception, nous observons l'objet, nous rencontrons son existence; mais l'objet ne nous est alors donné que d'un point de vue à la fois. Le propre de la perception, c'est que l'objet n'y paraît jamais que dans une série de profils; il n'est donc vu que d'une certaine façon qui appelle et exclut à la fois une infinité d'autres points de vue. Dans ce cas, on doit apprendre les objets, c'est-à-dire en faire le tour et multiplier sur eux les coups de sonde possibles. Dès lors, cette unité synthétique d'une multiplicité d'apparences qu'est la perception, n'épuise jamais l'objet; celui-ci déborde la conscience. En outre, dans la perception, l'élément proprement représentatif correspond à une certaine passivité de la conscience ${ }^{20}$. Il en est autrement de la conscience imageante. L'image est une certaine façon qu'a la conscience de se donner un objet et non un simulacre de l'objet dans la conscience; elle n'est rien d'autre qu'un rapport de la conscience avec l'objet. Cependant, différemment de la perception, la conscience imageante est un acte synthétique qui livre tout l'objet, parce que cette conscience est spontanée et créatrice : dans l'image, l'élément proprement représentatif est le produit d'une activité consciente. Il s'ensuit nécessairement que l'objet en image n'est jamais rien de plus que la conscience qu'on en a ; en ce sens, la conscience imageante ne rencontre rien et n'apprend rien que ce qu'elle y a déjà mis. Voilà pourquoi, dans la conscience imageante, il y a une espèce de manque ontique, d'une part, et, d'autre part, le rapport de cette conscience à l'objet est un rapport de certitude.

Ainsi donc, dans le spectacle, l'acte de la conscience perceptive nous fait rencontrer un aspect de l'événement, tandis que celui de la conscience imageante va chercher son objet sur le terrain de la perception et ne livre que ce que la conscience y met : dans le premier cas, l'événement est affecté du caractère de réalité et peut avoir un sens riche et profond ; dans le second cas, l'événement est constitué en objet irréel pour mieux le voir

20. Cette passivité doit être comprise au sens d'une active réceptivité ; être passif, pour l'être humain, ce n'est pas demeurer totalement inerte : c'est ne pouvoir prendre l'initiative d'une action. 
et le posséder, mais souffre, pour ainsi dire, d'une pauvreté essentielle.

En outre, le spectacle est une vision du monde qui sollicite d'abord et avant tout le regard. Dans l'histoire occidentale de l'esprit humain, les époques où le rapport humain au monde semble avoir trouvé ses plus forts élans de rigueur ont été précisément celles où le regard avait reçu un rôle privilégié ; cette hégémonie du sens visuel, des Grecs jusqu'à nous, nous livre un monde objectivé comme une chose, où l'expérience des autres sens n'est que postérieurement inscrite, en quelque sorte, dans cette image optique. Pourquoi cet intérêt exclusif accordé au regard ? Le regard est le sens de la distanciation et de l'objectivation, mais aussi de l'appropriation sans la confusion du contact. Par le regard se révèlent à moi un lieu où je ne suis pas et un objet que je ne suis pas. Par le regard, j'accède à ce qui m'échappe et je prétends à ce qui n'est pas moi, sans compromission de tout mon être. En se projetant sur cet événement, le regard m'instruit de la séparation et de la différence entre moi et cet objet, devenant ainsi le signe de ma maîtrise de cet événement : celui-ci est ma visée, mon intention, mon projet, mon désir ; les choses n'ont d'autre rôle que d'obéir à la quête ou au projet de quête du regard.

En réalité, cette suprématie du regard est trompeuse. En effet, si le regard me donne lieu à l'objectivité, il m'écarte par contre du contact intime des corps que le désir exige : il n'est que l'image visible du désir. En outre, la mass-médiatisation audio-visuelle contribue à déformer ce rapport optique avec l'événement : celui-ci n'est plus ma visée et mon projet, mais le résultat du procès technologique des communications de masse qui me dit qui regarder et où regarder; toute la matière de l'événement est normalisée et substituée à un univers multiple de signes. La mass-médiatisation audio-visuelle ne donne ni à voir ni à comprendre l'événement dans sá spécificité historique, sociale et culturelle, mais le livre plutôt selon un code homogène, qui est tout à la fois une structure technique et une structure idéologique.

Ces développements trop sommaires peuvent cependant éclairer notre compréhension du spectacle sportif et les effets de 
celui-ci sur le spectateur. Si le spectacle sportif correspond à l'événement qui s'éloigne dans une représentation parce que le contenu événementiel est tout concentré dans le regard et la conscience optique, il devient alors une sorte d'inversion de la vie concrète. Dès lors, le rapport social entre les individus humains est un rapport médiatisé par des images. Certes, pour le spectateur du stade, la conscience perceptive qui-fait rencontrer réellement l'événement sportif, ou plutôt un aspect à la fois de cet événement ; sa conscience imageante s'emploiera à créer une symbolique de l'événement. Dans le cas du spectateur télévisuel, la conscience perceptive ne rencontre pas l'événement sportif directement, mais son médium : par l'image télévisuelle, le spectateur sportif passe ainsi de l'événement signifié à la consommation de cette image en tant que telle ; sa conscience perceptive ne saisit qu'une caricature d'événement et sa conscience imageante est contrainte à créer une symbolique dérivée. Dès lors, on peut comprendre que le rapport du spectateur à l'événement sportif est un rapport abstrait et mystifiable : c'est un rapport étranger à la rencontre et au dialogue ; en fait, ce rapport est ambivalent : il éclaire autant qu'il occulte.

Par la disponibilité de la conscience perceptive et la puissance créatrice de la conscience imageante, ainsi que par la surdétermination et la manipulation techniques du contenu événementiel, le sport devient objet de spectacle d'une façon directement proportionnelle à l'exaltation de la prouesse, de l'effort et de la discipline, à l'exhibition du mouvement géométrique des formes et des couleurs, à la manifestation de l'agressivité et de la violence exorcisées, à la mise en scène de la mort jouée. Dès lors, le spectacle sportif séduit par son évidence et sa transparence, par son esthétique chorégraphique, morale et rationnelle, par son tragique consenti et symbolisé. Par contre, le spectacle sportif est une occultation de l'événement sportif comme tel : d'une part, parce que seul le regard constitue le canal de réception et d'expression agissant sur le reste du corps qui, lui, réagit plutôt que d'agir, et, d'autre part, parce qu'à ce regard se substitue souvent l'œil multiple de la télévision qui normalise l'événement sportif et le systématise à travers son essence technique. Voilà pourquoi le spectateur sportif est amené à se projeter non pas dans la réalité, comme il le croit ou comme on le lui 
fait croire, mais dans l'image que lui offre le spectacle sportif ; il est ainsi invité à vivre par procuration un complexe de passions, de sentiments et d'idées. Dès lors, une certaine aliénation plus ou moins consentie du spectateur s'installe au profit de l'objet contemplé : plus il contemple, moins il agit ; plus il accepte de se reconnaître dans les images dominantes du besoin, moins il comprend sa propre existence et son propre désir.

\section{Conclusion}

Au terme de cette étude du corps sportif, nous tirerons ces quelques conclusions provisoires. D'une part, il nous apparaît que le corps humain devient plus intelligible lorsque nous le saisissons comme une organisation d'énergies plutôt que comme une substance étendue sans cesse confrontée à une raison autonomiste. Le concept de corps comporte plusieurs connotations et fait l'objet d'une pluralité de pratiques et de discours parce qu'il est d'abord vécu comme une réalité plurielle : il l'est comme structure morphologique, osseuse, organique et musculaire et comme système de fonctions ; il l'est comme schéma postural et sensorimoteur dans la singularité d'une histoire personnelle, comme foyer d'agressivité, de désir et de plaisir, comme relation avec autrui, comme symbole d'une culture déterminée ; comme " ouverture et carrefour du champ symbolique », comme force ou instrument de travail alors même qu'on en restaure les énergies pour le temps de labeur. C'est à propos de ce corps pluriel que la raison pure, formaliste et techno-industrielle, devenue sportive, prétend tenir un langage cohérent en exhibant un seul et même corps : le corps sportif.

Mais pourquoi, d'autre part, la raison a-t-elle recours au sport pour réhabiliter plus efficacement le corps ? À notre avis, c'est parce qu'un processus de modélisation du corps par le sport est plus intégratif que tout autre, dans la mesure où, après avoir puisé dans le système social global industriel et dans l'idéologie capitaliste les éléments stratégiques de sa constitution, il les organise, les associe aux données d'une corporéité à multiples facettes, récupérée selon les principes normatifs de la rationalité sportive. Dans cette optique, ainsi que nous avons essayé de le suggérer, la réalité sportive nous semble plus compréhensible si nous empruntons une approche dialectique et si nous la défi- 
nissons comme l'organisation de ce qui, dans le corps humain, est puissances physiques et organiques, pulsions agressives et libidinales, configurations et pratiques socio-culturelles, spectacularisation et mercantilisation du geste soumis aux canons de la performance, d'un style normalisé et d'une politique du prestige. Dès lors, le concept même de sport pourrait tout à la fois signifier une technique tant de l'activité physique et de la concentration psychologique que de l'organisation d'une agonistique axée sur des enjeux déterminés et canalisée dans un cadre de réglementations, une pratique sociale de loisir, une spectacularisation et une mercantilisation du geste travaillé et stylisé, un foyer de mythes aux résonnances idéologiques et éthiques. Le sport est donc une réalité polysémique, et c'est en tant que tel qu'il peut prétendre s'approprier le corps comme organisation d'énergies multiples.

Ainsi compris, le sport moderne peut bien être affirmé comme la forme dominante d'éducation du corps humain. Toutefois, ce corps, récupéré par la raison sportive, est-il encore un foyer authentique d'agressivité, de fantasmes et de désirs, le pivot de la conscience et l'expression de la parole ? N'est-il pas plutôt un corps remodelé selon les finalités du pouvoir de la raison, soumis au capital et à la machine, fonctionnalisé en valeur d'usage et valeur d'échange économique et symbolique, fait pour la consommation, un corps redécouvert sans doute, exalté même, mais pour devenir le plus beau et le plus éclatant des objets dans le contexte généralisé des autres objets ?

Si notre interprétation est pertinente, il faut dire que le processus de sportivisation du corps et l'homologie entre celuici et les objets entraînent des conséquences profondes. Non seulement cette récupération par la raison sportive du corps humain, au sens où nous avons tenté de le comprendre, conduit à sa réhabilitation qui, en réalité, est celle d'un corps devenu objet/ signe, mais, plus profondément encore, elle engage, à notre avis, des rapports spécifiques entre l'ordre des choses et celui de la pensée. Ainsi, par exemple, dans ce procès généralisé de sportivisation, c'est le monde même de l'être qui est atteint : il l'est dans ses rapports avec l'apparaître, avec l'avoir et avec le sujet lui-même ${ }^{21}$.

21. Ces considérations concluantes ne doivent pas laisser entendre que nous avons une connaıssance précise des concepts d'être, de paraître et d'avoir. La question de leur sens et 
D'une part, en effet, tout dans ce procès est spectacularisé, c'est-à-dire évoqué, provoqué, orchestré en images, en signes, en modèles consommables. Ce procès d'absorption de signes et par les signes tient l'apparence pour plus honorable que l'être. Or, lorsque tout s'abolit et s'uniformise dans le signe, il n'y a plus de distance ontologique entre l'être et l'apparence. De la même manière, l'être et l'avoir perdent leurs lignes de partage dans la mesure où celui-ci se substitue à celui-là ; l'être finit par refléter l'avoir ; celui-ci prend des allures d'essence de l'être ; la contradiction de l'être n'est plus possible. Par ailleurs, dans la mesure où l'ordre de l'avoir est celui de la propriété objectivante, il s'apparente à celui du paraître : l'objet convoité et approprié l'est davantage dans sa fonctionnalité de signe que dans sa matérialité. En fin de compte, c'est le sujet lui-même qui est touché par cet envahissement progressif du paraître et de l'avoir ; il en arrive même à se définir en fonction de ses manifestations et de ses acquisitions ; elles en constituent son identité.

Où trouver, d'autre part, une explication à ce glissement de l'être dans le paraître et l'avoir, sinon dans une démarche discursive et imageante de l'ordre du connaître, car c'est bien du connaître que doit partir toute entreprise de connaissance et d'action humaine ? Certes, l'ordre du connaître peut bien s'avérer original par rapport à celui de l'être, en raison de son principe, de son processus et de sa finalité ; il se confond cependant plus facilement avec l'ordre de l'avoir dans la mesure où son processus est acquisitif d'essences et où son activité est celle d'un pouvoir de réduction du réel à l'intelligible. Dès lors, il est tentant de prendre cette originalité initiale pour de l'autosuffisance et de l'autoréalisation. Mais n'est-ce pas là opter pour un idéalisme absolu qui, fondant la réalité des choses sur le concept, prend les abstractions pour la réalité même ? La révolution copernicienne de Kant est ici à l'œuvre : on suppose que les objets (le corps) doivent se régler sur la raison du sujet. C'est

de leur portée reste béante et toujours urgente. Il nous a semblé que c'est jusque là que nous entraîne une réflexion sur une prise en charge du corps par la raison sportive, et que pour bien comprendre ce processus de sportivisation du corps humain, il faut pousser l'analyse jusque dans les parages de l'ontologie. 
donc, en fin de compte, une critique de la raison elle-même qu'il faut mener et maintenir afin de la préserver de cette frénésie paranoïaque d'introduire l'idée d'ordre jusque dans le désordre ou l'ineffable - le corps comme tel reste toujours irréductible à sa représentation, même si elle seule peut le rendre intelligible - et de ne rien supporter qu'elle-même ; c'est l'autorité du concept qu'il faut abroger et son désensorcellement qu'il faut pratiquer. Sinon l'homme se prend aux pièges de son savoirpouvoir et des connivences du croire et du faire croire ; il se fait, selon la célèbre formule de Protagoras, « la mesure de toutes choses ». Mais, en réalité, cet homme n'est jamais en face de ses propres besoins et du propre produit de son travail ; il n'est même plus capable de perspective sur lui-même parce que devenu «immanent aux signes qu'il ordonne ${ }^{22}$. Réduit à sa perception sensible du monde, à sa dimension de pragmaticien, à son pouvoir acquisitif, à la puissance de son efficacité, il est une mesure elle-même mesurée par cette dernière fiction conductrice de sens, de valeurs et d'actions qu'est la sportivisation du corps humain parvenu au statut paradigmatique de corps sportif.

Département de philosophie Université d'Ottawa

22. Jean BAUDRILLARD, La société de consommation, Paris, Gallimard, 1970, p. 309. 